www.jmscr.igmpublication.org

Impact Factor (SJIF): 6.379

Index Copernicus Value: 79.54

ISSN (e)-2347-176x ISSN (p) 2455-0450

crossrefDOI: https://dx.doi.org/10.18535/jmscr/v6i9.58

Journal Of Medical Science And Clinical Research

\title{
Ruptured interstitial ectopic pregnancy and its management: A Case Report
}

\author{
Authors \\ Dr Vinodkumar Suresh Basavaradder ${ }^{1^{*}}$, Dr Ratana Usham ${ }^{2}$ \\ ${ }^{1}$ (PGT), Obstetrics and Gynecology, JNIMS, Imphal, Manipur \\ ${ }^{2}$ Associate Professor JNIMS Imphal, India
}

\begin{abstract}
Background: Cornual or Interstitial Ectopic pregnancy is a rare, catastrophic and the leading cause of maternal mortality in first trimester of pregnancy.

Result: A 37 year-old para-4-0-2-4 woman presenting to gynaecological emergency department with h/o 2 $1 / 2$ months of amenorrhoea and complaints of spotting PV, pain abdomen and vomiting since 1 day. Clinically diagnosis of ruptured ectopic pregnancy was made and confirmed using USG. Intraoperatively right cornual ruptured ectopic pregnancy was found. Right cornuostomy with repair with left tubal ligation was done.

Conclusion: The diagnosis of an interstitial ectopic pregnancy is usually difficult and delayed resulting in high morbidity and mortality. Our patient treated by right cornuostomy with repair and left tubal ligation.

Keywords: Ectopic pregnancy, Cornual pregnancy, Cornuostomy.
\end{abstract}

\section{Introduction:}

Ectopic pregnancy is the leading cause of maternal mortality in first trimester of pregnancy. The incidence of ectopic pregnancy increasing as increase in incidence of assisted reproduction techniques and STD. Ectopic pregnancy in interstitial part of fallopian tube is rare and is also catastrophic $^{1}$. Interstitial ectopic pregnancy defined as gestation sac developing in the uterine part of fallopian tube. The interstitial part of the fallopian tube contains more vascularized and muscular tissue. Due to this vascular and connective tissue support and anatomic localization, diagnosis and treatment is usually late and rupture takes place later during pregnancy term and causes massive Haemorrhage. This condition difficult to diagnose both clinically and sonographically.

\section{Case Report}

A 37 year-old para-4-0-2-4 woman presenting with complaints of spotting PV, pain abdomen and vomiting since 1 day following a history of 2 $1 / 2$ months of amenorrhea and past $\mathrm{h} / \mathrm{o}$ twice $\mathrm{D} \& \mathrm{C}$, with no h/o previous ectopic pregnancy, pelvic inflammatory disease or the utilization of assisted reproductive techniques was referred to emergency ward. On examination PR was $90 \mathrm{bpm}$, BP was $90 / 60 \mathrm{mmHg}$ and initial $\mathrm{Hb} \%$ was 6.8 $\mathrm{g} / \mathrm{dl}$.A UPT performed was found to be positive. Ultrasound examination revealed an empty uterine cavity with heteroechoic lesion in right adnexa with probe tenderness and fluid in pouch of douglas. This raised the possibility of ruptured ectopic pregnancy. Our patient was managed by Exploratory Laparotomy, where the ectopic gestation sac located in the cornual portion of the 
ruptured right fallopian tube with around $2 \mathrm{~L}$ of blood clots in peritonium was identified. The primary surgical procedure undertaken were as follows, exploratory laparotomy and cornuostomy with repairwith left tubal ligation was performed

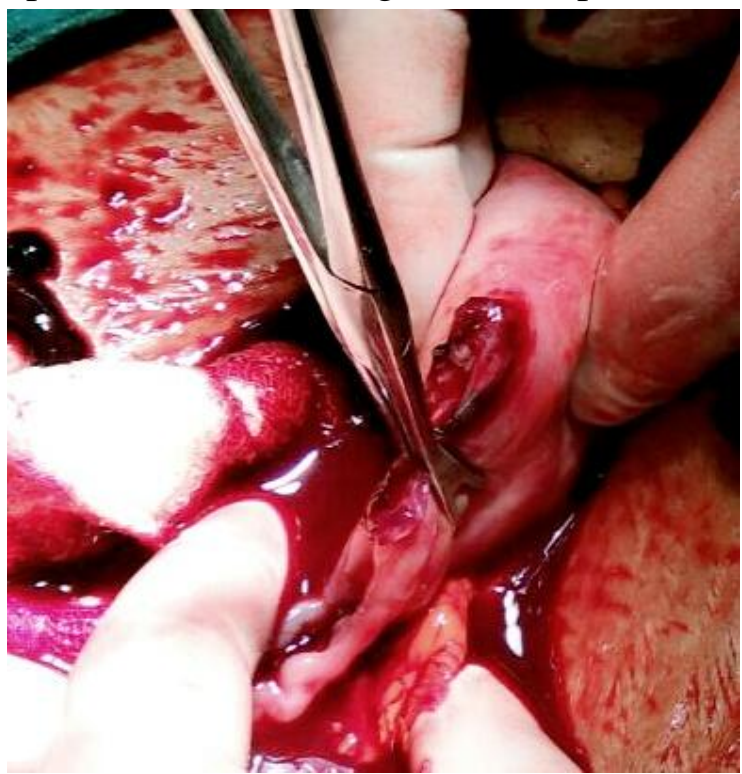

Figure 1: Intraoperative view of right ruptured and 3 units of PRBC transfused units intraoperatively and 1 unit post operatively. Patient withstood the procedure well without any intraoperative or postoperative complications.

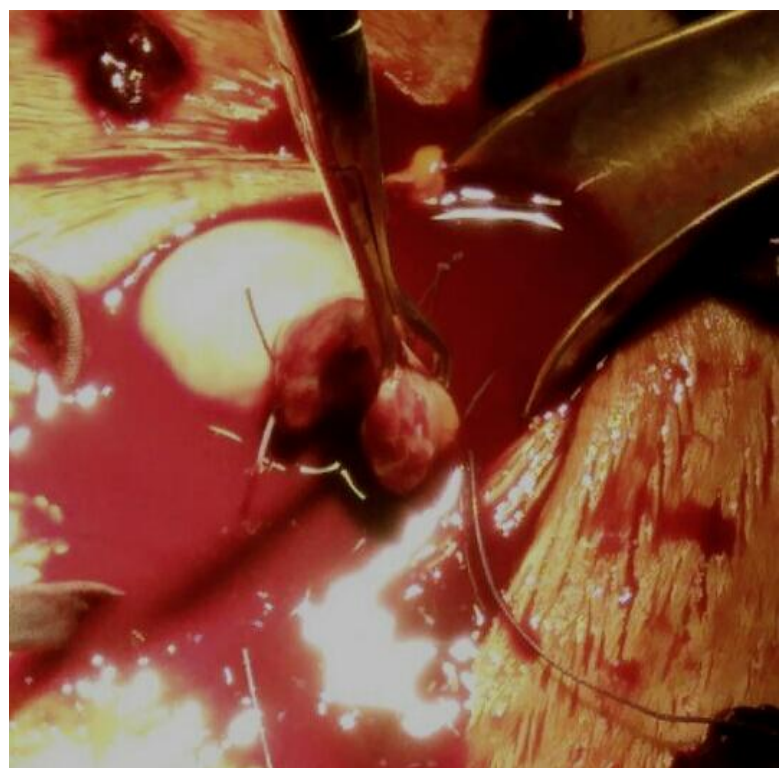

Figure 2: Intraoperative view of cornuostomy interstitial ectopic pregnancy

\section{Discussion}

Ectopic pregnancy is the implantation of blastocyst outside the uterine cavity, ampullary part of fallopian tube being the most common site and other sites include ovaries, abdominal cavity and cervix. Its incidence showing increasing trend due to increasing risk factors like Pelvic infections, artificial reproductive techniques, previous tubal surgeries and peritubal adhesions following salpingitis, appendicitis, endometriosis may cause tubal kinking and narrowing of the lumen and thereby increase the risk of tubal pregnancy ${ }^{2}$. Ankum et $\mathrm{al}^{2}$ reported that Prior tubal surgery, either to restore patency or to perform sterilization, confers the highest risk of obstruction.

Outcome of ectopic pregnancy include tubal rupture, tubal abortion and pregnancy failure with resolution. Usually the tubal ruptures occurs early weeks of gestation in isthmic part, ampullary part of fallopian tube as the fallopian tube lacks submucosal layer compared to cornual (interstitial) ectopic pregnancy which usually occurs before 12 weeks as reported by Tulandi et. $\mathrm{al}^{3}$.

The rupture of interstitial ectopic pregnancy causes torrential bleeding as there is anastomosis of uterine and ovarian arterial branches ${ }^{4}$ and is one of the life-threatening situations like shock, hemoperitoneum in women in early pregnancy compared to other sites of ectopic pregnancy.

As there is higher risk of maternal mortalilty, early diagnosis and management is indicated but the diagnosis of ectopic pregnancy usually difficult and delayed ${ }^{5}$.

The diagnosis of ectopic pregnancy can be made clinically in women presenting with history of amenorrhoea, PV bleeding, pain abdomen, shock and serial beta hCG measurements and radiologically by the presence of an eccentrically located gestation sac with incomplete or asymmetric myometrial tissue, $<5 \mathrm{~mm}$ in thickness, is a highly suggestive but nonspecific indicator of interstitial pregnancy ${ }^{1}$. The presence of an echogenic line between the gestation sac and the endometrial cavity, also known as the 
interstitial line sign, is highly sensitive and specific for interstitial ectopic pregnancy ${ }^{6}$. The discriminatory beta-hCG above $1500 \mathrm{mIU} / \mathrm{ml}$ levels, failure to visualize a uterine pregnancy by transvaginal ultrasound indicates with high reliability that the pregnancy is either ectopic or nonviable. If the initial beta hCG below this level, serial beta $\mathrm{hCG}$, repeat transvaginal ultrasound ${ }^{7}$ indicated only in hemodynamically stable patient and also distinction between nonliving uterine versus an ectopic pregnancy can be made by uterine curettage, or in some cases, by endometrial biopsy. Serum progesterone level $>25 \mathrm{ng} / \mathrm{ml}$ excludes ectopic pregnancy ${ }^{8}$.

Treatment options for interstitial ectopic pregnancy include medical methods like local injection or systemic therapy with methotrexate, local injection of potassium chloride indicated only in hemodynamically stable patients and who can follow up with serum beta hCG, but practically rarely used in interstitial ectopic pregnancy. The best medical treatment regimen for interstitial pregnancy remains unknown.

Evidence of a haemorrhagic ectopic pregnancy is an indication for laparotomy and the traditional treatment of interstitial pregnancy is surgical methods like cornual resection, hysterectomy, in cases with severely damaged uteri. Nowadays increasingly more conservative approaches are being used, such as cornuostomy instead of cornual resection and hysterectomy, as well as laparoscopy in place of laparotomy. Cornual resection may be assisted by direct injection of vasoconstrictive agents such as diluted vasopressin in order to reduce blood loss. This conservative surgical measure appears to have no negative impact on the outcome of subsequent pregnancies. Other surgical option include Salpingostomy , Salpingotomy, Salpingectomy. In our case report the patient came with ruptured ectopic pregnancy which as dignosed intraoperatively as ruptured right interstitial ectopic pregnancy and managed right cornuostomy with left tubal ligation.

\section{Conclusion}

The diagnosis of an interstitial ectopic pregnancy is usually difficult and delayed resulting in high morbidity and mortality, hence early diagnosis and treatment is essential. Our patient treated by right cornuostomy with repair and left tubal ligation, recovered with no post operative complications.

\section{Reference}

1. Wood C, Hurley V. Ultrasound Diagnosis and Laparoscopic Excision of an Interstitial Ectopic Pregnancy.Aust $\mathrm{N}$ Z J Obstet Gynaecol.1992;32:371-2.

2. Ankum WM, Mol BWJ, Van der Veen F, et al: Risk factors for ectopic pregnancy: A meta-analysis. Fertil Steril 65:1093, 1996 [PMID: 8641479]

3. Tulandi T, A1-Jaroudi D: Interstitial pregnancy: Results generated from the Society of Reproductive Surgeons. Obstet Gynecol 103:47, 2004 [PMID: 14704243]

4. Dorfman SF, Grimes DA, Cates W Jr, et al: Ectopic pregnancy mortality, United States, 1979 to 1980: Clinical aspects. Obstet Gynecol 64:386, 1984 [PMID: 6462568]

5. Maliha WE, Gonella P, Degnan EJ. Ruptured interstitial pregnancy presenting as an intrauterine pregnancy by ultrasound. Ann Emerg Med. 1991;20:910-2.

6. Ackerman TE, Levi CS, Dashefsky SM, Holt SC, Lindsay DJ. Interstitial line: Sonographic finding in interstitial (cornual) ectopic pregnancy. Radiology. 1993;189:83-7.

7. Barnhart KT, Simhan H, Kamelle SA: Diagnostic accuracy of ultrasound, above and below the beta-hCG discriminatory zone. Obstet Gynecol 94:583, 1999 [PMID: 10511363]

8. Pisarska MD, Carson, Buster JE: Ectopic pregnancy. Lancet 1998; 351:1115. 